\title{
PORTAL DE PERIÓDICOS UFSC: IMPLANTAÇÃO DO SISTEMA DE CHAMADOS
}

José Paulo Speck Pereira ${ }^{1}$, Lucia da Silveira ${ }^{1 *}$, Juliana Aparecida Gulka ${ }^{1}$, Gabriel Araldi Walter ${ }^{1}$, Alexandre Pedro de Oliveira ${ }^{1}$, Mirna Cassettari Saidy ${ }^{1}$

1 Universidade Federal de Santa Catarina - Sistema de Bibliotecas - Portal de

Periódicos Científicos - Florianópolis (SC) - Brasil

*Autor correspondente: luciadasilveiras@gmail.com

\author{
Como citar: \\ PEREIRA, José P. S. et al.. Portal de periódicos ufsc: implantação do sistema de chamados. In: \\ WORKSHOP DE EDITORAÇÃO CIENTÍFICA, 9., 2016, São Paulo. Anais... São Paulo: \\ Associação Brasileira de Editores Científicos, 2016. p. 54-59 \\ DOI: http://dx.doi.org/10.21452/wec.IXwec.2016.0011
}

\section{RESUMO}

Retrata a inovação de processo do Portal de Periódicos da Universidade Federal de Santa Catarina (UFSC) por meio da implantação do sistema de chamados para o atendimento às equipes editoriais. Utilizou a abordagem metodológica de estudo de caso descritivo. Aponta como resultado as vantagens e limitações do uso do sistema pela equipe do Portal. Conclui que a solução tecnológica proporcionou uma gestão mais transparente, padronizada e eficiente.

\section{INTRODUÇÃO}

A inovação nos serviços públicos tem o potencial de economizar recursos (o 'dinheiro de todos', obtidos pelos impostos), reorganizar equipes e processos de trabalho, adequar-se às novas dinâmicas da sociedade e até elevar a qualidade de vida de comunidades. A inovação para organizações é uma mudança estratégica na criação de um novo produto, renovação de um processo, melhorando sua produtividade ou desempenho dos bens, serviços e processos. (MANUAL DE OSLO, 2004). Por isso, entende-se que a abertura à mudança e à inovação deve ser uma constante nas instituições governamentais.

As práticas inovadoras de gestão podem harmonizar-se com os princípios legais que regem a administração pública. Os servidores públicos devem conduzir suas ações a partir de cinco princípios fundamentais, previstos na Constituição (artigo 37): a legalidade (atender somente o que está em lei), impessoalidade (atuar sob o interesse público e tratar igualmente as pessoas), moralidade (atuar com bases éticas), eficiência (melhor utilização dos recursos disponíveis) publicidade (a regra é disseminar, a exceção é a confidencialidade da informação) (CHIAVENATO, 2014). Em um mundo com mudanças constantes, a administração direta e indireta dos governos deve abrir-se à inovação para cumprir sua missão, pois caso contrário há a possibilidade de tornar-se desconectada das necessidades da população, ou mesmo obsoleta.

O objetivo deste trabalho, portanto, é retratar um processo de inovação do Portal de Periódicos da Universidade Federal de Santa Catarina (UFSC), especialmente as soluções empregadas nas demandas de atendimento ao público, que proporcionaram 
uma gestão mais transparente, padronizada e eficiente. As próximas seções descrevem o contexto da UFSC e do Portal, seguidas dos procedimentos metodológicos, resultados e considerações finais.

\section{PORTAL DE PERIÓDICOS UFSC}

O Portal de Periódicos UFSC é uma iniciativa institucional, com quase 10 anos de história, que garante o suporte aos seus periódicos científicos em acesso aberto, com o objetivo de promover seu acesso, visibilidade, qualificação e preservação.

Foi fundado pelo Departamento de Ciência da Informação (CIN), que desde o início adotou o Sistema Eletrônico de Editoração de Revistas (SEER), tradução brasileira do software Open Journal System (OJS). Este sistema padroniza e automatiza o fluxo editorial e o gerenciamento de periódicos científicos. Em 2008, a gestão do Portal passou à Biblioteca Universitária (BU). Em 2016, o Portal conta com a presença de 44 periódicos científicos.

O Portal conta com um Conselho Consultivo e Deliberativo (CCD), composto por representantes provenientes de diferentes departamentos da UFSC, que atuam na tomada de decisões com impacto a nível institucional - entre elas, a análise minuciosa de solicitações de inclusão de novos periódicos no Portal.

A equipe gestora do Portal contribui com a gestão técnica, institucional e operacional, e é composta por três bibliotecários, um auxiliar administrativo e uma revisora de textos, além da parceria e suporte permanente da Superintendência de Governança Eletrônica e Tecnologia da Informação e Comunicação (SeTIC) e do Laboratório de Periódicos Científicos ${ }^{1}$.

A equipe é qualificada e estuda de forma contínua as tendências da comunicação científica e os princípios de acesso aberto, oferecendo aos editores serviços personalizados. O esforço na melhoria dos serviços é reconhecido entre os pares: membros do Portal ministram palestras em eventos da área, e frequentemente bibliotecários e outros profissionais de diferentes estados brasileiros contatam a BU para solicitar consultoria. O Portal de Periódicos UFSC tornou-se uma referência nacional, ocupando o segundo lugar no ranking Top Portals elaborado pela Webmetrics (2016).

\section{PROCEDIMENTOS METODOLÓGICOS E A IMPLANTAÇÃO DO SISTEMA DE CHAMADOS NO PORTAL DE PERIÓDICOS UFSC}

O presente trabalho é caracterizado como um estudo de caso descritivo, pois retrata um acontecimento: a implantação do sistema de chamados em um ambiente específico e real (Portal de Periódicos UFSC). Para Yin (2014, p. 244) "o estudo de caso descritivo tem como propósito descrever um fenômeno (o "caso") em seu contexto do mundo real."

Em 2014, com a mudança da coordenação do Portal, percebeu-se a necessidade de uma maior sistematização em todas as atividades realizadas, de forma a evitar a descontinuidade das boas práticas, independentemente das trocas de cargos ou mudanças na composição da equipe.

A busca pela solução, foi orientada pela gestão da informação e tecnologia da informação, enquadrando-se no objetivo proposto que trata da implantação de um sistema de atendimento online, também denominado 'sistema de chamados' ou help desk. O uso do sistema permitiria o controle dos atendimentos realizados, e o acompanhamento das demandas de forma colaborativa pela equipe.

O processo de implantação do sistema de chamados iniciou em 2015 e foi realizado pela equipe do Portal em conjunto com a SeTIC. As etapas de implantação estão 
a) Reunião com a SeTIC: nessa etapa foram esclarecidas as funcionalidades do uso do sistema de atendimento. Vistas as opções que o sistema permitia, foi solicitado a equipe do Portal o mapeamento dos serviços oferecidos, organizados por meio de uma hierarquia dividida em categoria principal e secundária. Em outras palavras, foi necessário a categorização dos serviços do Portal para criação da interface do formulário de solicitação de atendimento a ser utilizado pelos usuários.

b) Mapeamento de serviços: o mapeamento foi realizado por meio da sistematização das demandas dos editores, 0 alinhamento de algumas exigências/recomendações de indexadores e as atividades já desempenhadas pela equipe do Portal.

c) Capacitação da Equipe: nesta fase, a equipe aprendeu a fazer um chamado (visão do solicitante), como responder (perspectiva do atendente) e como analisar as demandas por meio dos relatórios disponíveis no sistema de chamados.

Finalizadas estas etapas, em fevereiro de 2015, o Portal passou a operar e incentivar o uso do sistema de chamados, em substituição a mensagens trocadas por e-mail.

\section{USO DO SISTEMA DE CHAMADOS: RESULTADOS}

O primeiro resultado evidente foi a descrição clara de todos os serviço ofertados pelo Portal de Periódicos UFSC. A equipe compreendeu as suas atribuições bem como as contingências impostas pelas limitações de recursos, o que permitiu posicionar mais estrategicamente o Portal no contexto da comunicação científica. Em 2016, os serviços oferecidos são:

1) hospedagem de periódicos e assessoria na migração dos dados;

2) assessoria em: a) indexação em bases de dados nacionais e internacionais,

b) ética editorial, c) sistema de editoração, d) processo de avaliação por pares,

e) fluxo editorial, entre outros;

3) análise personalizada para as necessidades do periódico;

4) revisão estrutural de novas edições com a conferência técnica e gramatical dos metadados;

5) atribuição do Digital Object Identifier (DOI);

6) divulgação científica por meio das redes sociais.

Uma vez instalado e pronto para uso, as equipes editoriais dos periódicos foram comunicadas e capacitadas gradualmente. É necessário esclarecer que o sistema de chamados é intuitivo, não necessitando de muitas instruções para sua utilização.

A interface disponível ao público tem um formato de formulário, disponível a partir do link <atendimento.periodicos.ufsc.br>. Basta o usuário preenchê-lo. Mas se prefererir, poderá enviar mensagem para o e-mail <adminportal.bu@contato.ufsc.br> que um chamado dentro do sistema é criado automaticamente. Na figura 1 é possível observar a interface do sistema pela perspectiva do usuário, via endereço eletrônico na web.

Figura 1: Sistema de chamados - perspectiva do usuário

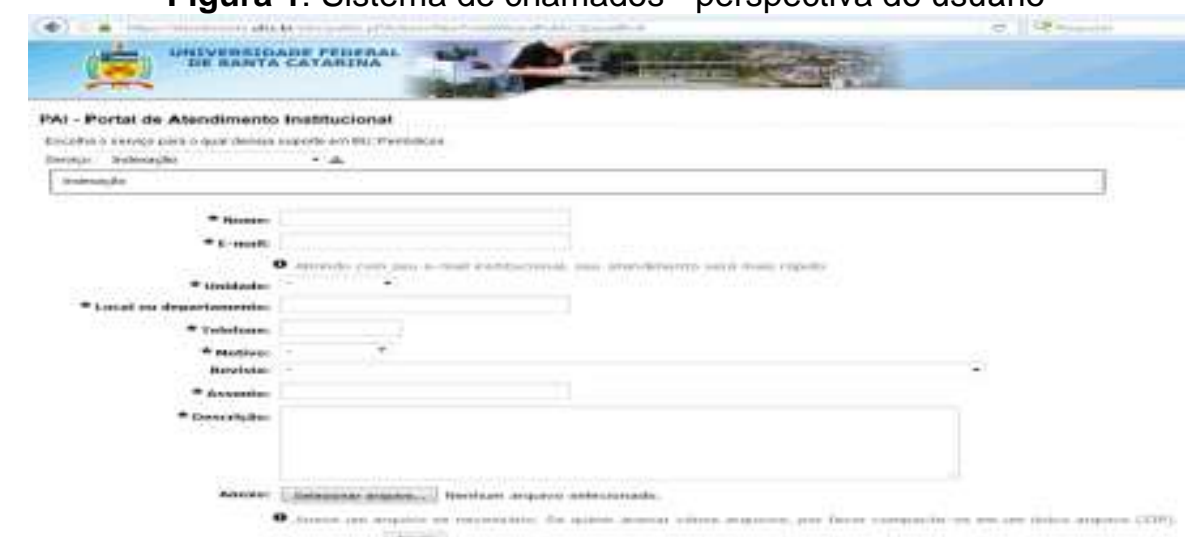


Em resposta à solicitação do usuário, o sistema automaticamente encaminha um e-mail para o solicitante informando-o do número do ticket ${ }^{2}$ para acompanhar 0 atendimento. O chamado então entra na fila de trabalho visível à todos os atendentes do Portal (figura 2), que realizam atendimentos conforme a sua competência e a ordem de chegada.

Figura 2: Sistema de chamados - perspectiva atendente

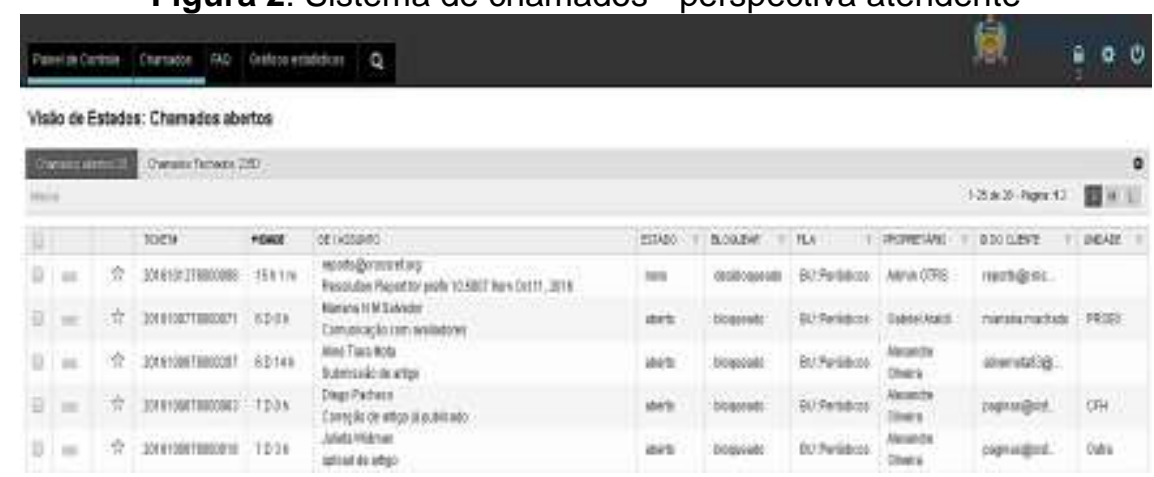

Com o uso do sistema de chamados, o Portal contabilizou 1336 solicitações em 2015. De janeiro a setembro de 2016, foram contabilizados 924 (dados gerados pelo sistema). Salienta-se que um mesmo chamado (solicitação) pode agrupar várias mensagens. A figura 3 mostra o crescimento dos atendimentos em 2016 e a tendência de maior fluxo de atendimento nos meses de publicação de nova edição dos periódicos, de dezembro a janeiro e junho a agosto.

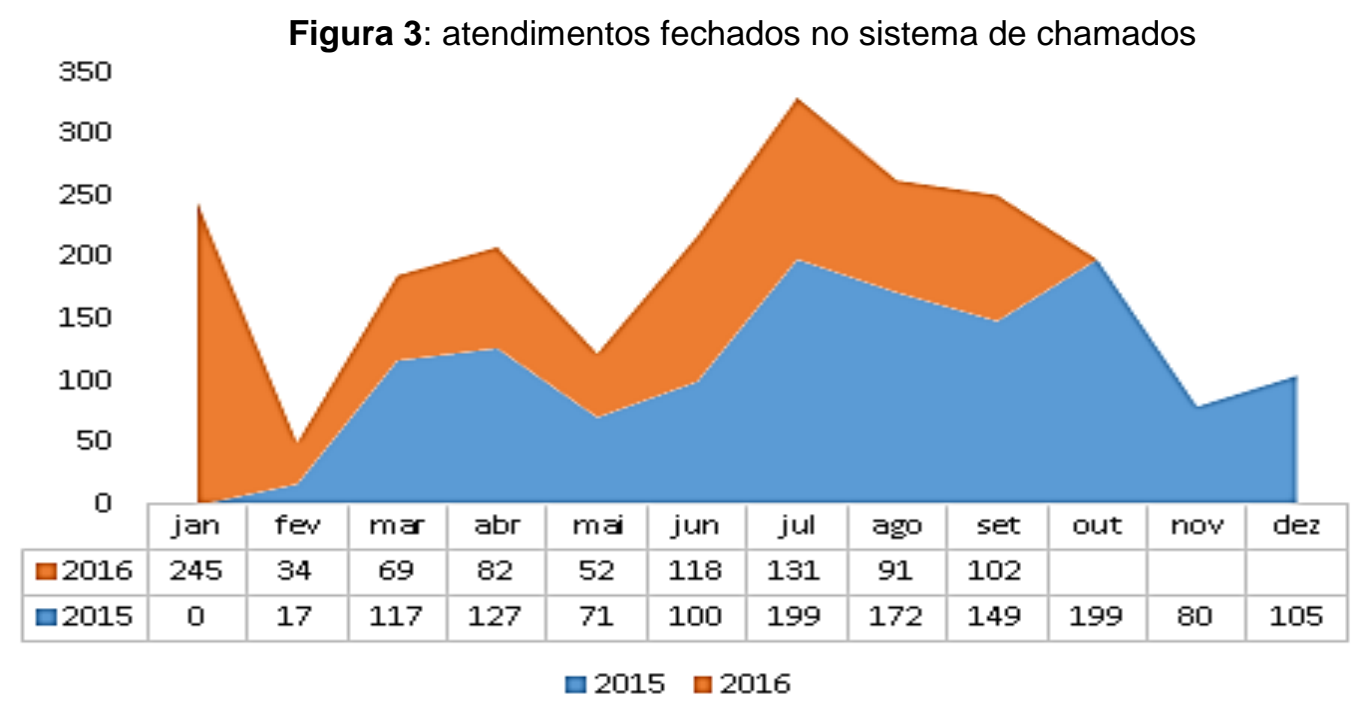

Desde a implantação do sistema, a equipe conseguiu identificar vantagens e limitações de seu uso, que são descritas na sequência:

Vantagens:

a) Transparência no processo e compartilhamento de informações principalmente na visão interna - em que todos os membros da equipe podem acompanhar e contribuir com os atendimentos; o acesso aos atendimentos permitiu o compartilhamento de informações da equipe do Portal, refletindo na melhoria do atendimento ao editor.

b) Organização dos atendimentos - os chamados abertos ficam em uma fila de espera; os já respondidos em outra, onde aguardam para serem fechados; 
c) Otimização do tempo de atendimento - mediante o uso de respostas automáticas e manuais na resolução de problemas frequentes.

d) Diminuição do uso de e-mails - principalmente o uso dos e-mails pessoaisinstitucionais substituído pelo e-mail institucional direcionado para o sistema de chamados.

e) Gradual padronização de procedimentos - por meio da identificação de demandas frequentes, comuns a vários periódicos, foram elaborados manuais. Os manuais são incluídos no módulo do sistema de chamados nomeado como perguntas frequentes, que possibilita tanto o acesso público pelos usuários quanto a sua inclusão nas respostas às solicitações.

f) Melhor distribuição de atividades - com o uso dos chamados, as atividades foram distribuídas pelas competências individuais de cada servidor da equipe do Portal.

Limitações:

a) Mudança de cultura - no primeiro ano de uso do sistema de chamados alguns editores resistiram no uso dessa tecnologia. Como forma de contornar o recebimento de demanda por e-mail pessoal-institucional, a equipe do Portal respondia a solicitação via sistema de chamados ao solicitante.

b) Edição da resposta - o sistema não salva a versão de rascunho.

c) Elaboração de estatísticas - o sistema apresenta no menu o item gráficos estatísticos com sete opções de relatórios, entretanto, não existem opções puramente numéricas para a quantificação dos resultados, apenas os gráficos para a visualização. Ainda, as opções de geração de gráficos que utilizam o filtro "por serviço" agrupam todos os serviços atendidos via sistema de chamados por toda a Universidade, ficando difícil realizar a estatística de forma categorizada. Além disso, no módulo de perguntas frequentes não indica estatísticas de uso.

\section{CONSIDERAÇÕES FINAIS}

O presente trabalho descreveu o processo de implantação do sistema de chamados utilizado para atendimento online do Portal de Periódicos UFSC. Tal iniciativa é considerada uma inovação, permitindo de forma estratégica a renovação de um processo, culminando em melhorias tanto para a equipe interna como para os usuários atendidos, aliando a esse processo os princípios fundamentais da administração pública, sobretudo em relação à eficiência.

A solução empregada no atendimento dos usuários do Portal foi baseada no uso da tecnologia da informação, a partir de um software livre. O uso do sistema permitiu o acompanhamento dos chamados recebidos por toda a equipe que atua no Portal de Periódicos UFSC, resultando em uma atividade mais colaborativa e com 0 compartilhamento das informações estratégicas e tomadas de decisões de uma forma mais sistematizada.

A gestão transparente das demandas, bem como sua padronização e organização refletiram em uma elevação da qualidade do atendimento prestado aos usuários, justificado por uma maior agilidade na resposta e no repasse de informações especializadas.

A utilização do sistema de chamados possibilitou também, que a equipe gestora do Portal percebesse as lacunas em relação às especificidades necessitadas pelas equipes editoriais, que nem sempre eram passíveis de serem resolvidas sem um encontro presencial. Dessa forma, para suprir essas questões, a equipe vem planejando a implantação de um Programa de Formação Continuada de Equipes Editoriais, previsto para ser lançado em 2017, inicialmente com a oferta de 7 módulos estruturantes, que contemplam desde questões mais técnicas (como o sistema de editoração, normas, etc.) a tendências e necessidades específicas (divulgação científica e marketing, editais de fomento, etc.).

Dessa forma, o Portal de Periódicos UFSC vem profissionalizando sua gestão, buscando atender sua missão de modo a oferecer um suporte seguro e prático às 
demandas de sua comunidade editorial, bem como soluções atentas aos desafios da comunicação científica.

\section{REFERÊNCIAS}

BRASIL. FINEP. Manual de Oslo: Proposta de Diretrizes para Coleta e Interpretação de Dados sobre Inovação Tecnológica. 2004. Disponível em:

$<$ http://www.finep.gov.br/images/a-finep/biblioteca/manual de oslo.pdf>. Acesso em: 13 out. 2016.

\section{UNIVERSIDADE FEDERAL DE SANTA CATARINA. PORTAL DE}

PERIÓDICOS UFSC. Disponível em: <https://periodicos.ufsc.br/>. Acesso em: 10 out. 2016.

. UNIVERSIDADE FEDERAL DE SANTA CATARINA. UFSC em números: 2006 a 2015. 2016. Disponível em: <https://goo.gl/0LGVdw>. Acesso em: 10 out. 2016.

CYBERMETRICS LAB. Ranking web of repositories. 2016. Disponível em: $<$ http://repositories.webometrics.info/en/Latin America/Brazil>. Acesso em: 10 out. 2016.

YIN, Robert K. Estudo de caso: planejamento e métodos. 5. ed. Porto Alegre: Bookman, 2015. 